\title{
Resultados de la ablación por radiofrecuencia en el tratamiento de carcinoma hepatocelular en Veracruz, México
}

\author{
Radiofrequency ablation results in hepatocellular \\ carcinoma treatment in Veracruz, Mexico
}

Gustavo Martínez-Mier, ${ }^{*}$ Alma Yrani Escobar-Ríos, ${ }^{*}$ Sergio Esquivel-Torres, ${ }^{\ddagger}$ Iván Eliud Casanova-Sánchez, ${ }^{\S}$ Alonso Heriberto Ramírez-Sánchez

\section{Palabras clave: Carcinoma hepatocelular, ablación por radiofrecuencia, supervivencia, índice de masa corporal. \\ Keywords: Hepatocellular carcinoma, radiofrequency ablation, survival, body mass index.}

\footnotetext{
* Departamento de Investigación, Unidad Médica de Alta Especialidad (UMAE) No. 189 "Adolfo Ruíz Cortines", Instituto Mexicano del Seguro Social, Veracruz, México. ₹ Departamento de Cirugía Oncológica, Hospital de Alta Especialidad de Veracruz, Servicios de Salud de Veracruz, Veracruz, México.
}

\section{RESUMEN}

Introducción: El carcinoma hepatocelular es el tumor primario hepático maligno más frecuente. Sólo 15\% de los pacientes será sometido a resección quirúrgica o trasplante, por lo que la ablación local es un tratamiento alternativo con ventajas. Objetivo: Evaluar la eficacia de la ablación por radiofrecuencia en nuestra población. Material y métodos: Estudio retrospectivo de pacientes con carcinoma hepatocelular sometidos a ablación por radiofrecuencia; se investigaron variables sociodemográficas, del tumor, estadio (Child-Pugh, OKUDA, BCLC, CLIP, MELD y ALBI), uso de sorafenib y supervivencia. Resultados: Se analizaron 16 pacientes (edad media 71.25 años) con patología concomitante en $87.5 \%$. El 62.5\% de los pacientes padeció cirrosis, 56.3\% Child-Pugh A y 6.3\% B. El 62.5\% fueron OKUDA I, 37.5\% estadio BCLC A, 56.3\% BCLC B y $6.3 \%$ BCLC C; $56.3 \%$ se estadificó CLIP 0, MELD $8.44 \pm 2.15$ y ALBI $-2.54 \pm 0.42$. El tamaño tumoral fue $5.81 \pm 2.81 \mathrm{~cm}$ y sorafenib en $25 \%$. La supervivencia media fue 37.7 meses, supervivencia al año $58.5 \%$ y a cinco años $23.4 \%$. El índice de masa corporal se asocia a baja supervivencia $(\mathrm{p}=0.031)$. Conclusión: Nuestro estudio indica que la ablación por radiofrecuencia se usa en pacientes con carcinoma hepatocelular en estadios variados $y$ en ocasiones en tumores mayores a $5 \mathrm{~cm}$, haciendo que la supervivencia sea menor a otros estudios.

\section{ABSTRACT}

Introduction: Hepatocellular carcinoma (HCC) is the most frequent malignant primary liver tumor. Only $15 \%$ of patients will undergo surgical resection or transplantation, thus local ablation is an important treatment with many advantages. Objective: To evaluate the effectiveness of radiofrequency ablation in our population. Material and methods: Retrospective study of patients with hepatocellular carcinoma undergoing radiofrequency ablation, analyzing sociodemographic variables, tumor relate, stage (Child-Pugh, OKUDA, BCLC, CLIP, MELD and $A L B I$ ), use of sorafenib and survival. Results: Sixteen patients (average age 71.25 years) with concomitant pathology in $87.5 \%$, were analyzed. $62.5 \%$ of the patients suffered cirrhosis, $56.3 \%$ Child-Pugh A and 6.3\% B. 62.5\% of patients were OKUDA I. $37.5 \%$ with BCLC A, $56.3 \%$ BCLC B and 6.3\% BCLC C. $56.3 \%$ were CLIP 0. Average $M E L D 8.44 \pm 2.15$ and average $A L B I-2.54 \pm 0.42$. Tumor size was $5.81 \pm 2.81 \mathrm{~cm}$ and use of sorafenib in $25 \%$. Average survival was 37.76 months, survival rate $58.5 \%$ at one year and $23.4 \%$ at five. Low body mass index was associated with low survival ( $p=0.031)$. Conclusion: Our study indicates that radiofrequency ablation is used in patients with hepatocellular carcinoma in various stages and sometimes in tumors $>5 \mathrm{~cm}$, making survival shorter than other studies.

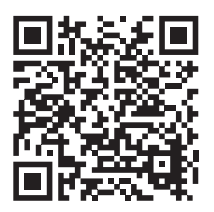

Citar como: Martínez-Mier G, Escobar-Ríos AY, Esquivel-Torres S, Casanova-Sánchez IE, Ramírez-Sánchez AH. Resultados de la ablación por radiofrecuencia en el tratamiento de carcinoma hepatocelular en Veracruz, México. Cir Gen. 2021; 43 (1): 23-29. https://dx.doi.org/10.35366/103910 


\author{
§ División de Radiología \\ Intervencionista, \\ Departamento \\ de Radiología e \\ Imagen Molecular, \\ Instituto Nacional de \\ Ciencias Médicas y \\ Nutrición "Salvador \\ Zubirán”, Ciudad de \\ México, México. \\ " Departamento de \\ Cirugía General, \\ Unidad Médica de \\ Alta Especialidad \\ (UMAE) No. 189 \\ "Adolfo Ruíz Cortines", \\ Instituto Mexicano \\ del Seguro Social, \\ Veracruz, México. \\ Recibido: 28/11/2019 \\ Aceptado: 29/11/2021
}

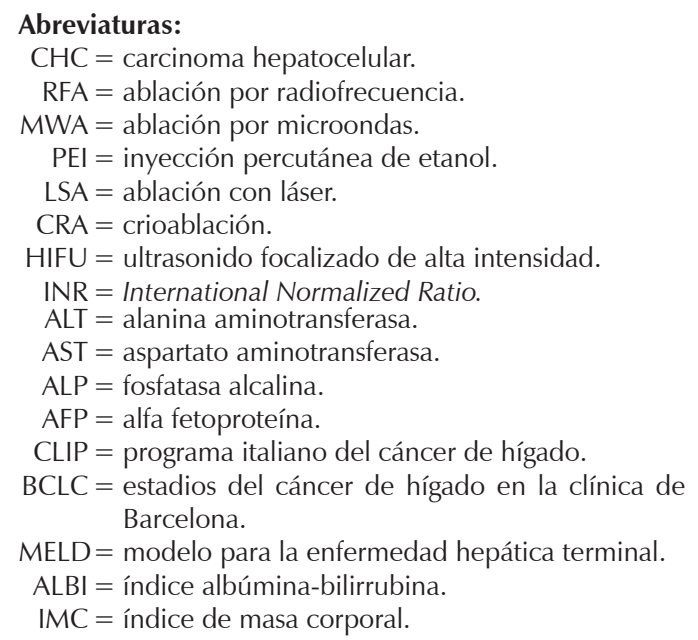

\section{INTRODUCCIÓN}

$\mathrm{E}^{\prime}$ carcinoma hepatocelular $(\mathrm{CHC})$ es el tumor primario hepático maligno más frecuente en el mundo. ${ }^{1}$ Es la quinta neoplasia más común en hombres y la octava en mujeres; representa la tercera causa de mortalidad relacionada con cáncer en población masculina. ${ }^{2}$ Los tratamientos del CHC incluyen trasplante de hígado, resección quirúrgica, y métodos de ablación local. El trasplante hepático es limitado, dada la escasez de donantes. ${ }^{3}$ Por desgracia, sólo el 5-15\% de los pacientes recientemente diagnosticados de $\mathrm{CHC}$ podrá ser sometido a una resección potencialmente curativa o a un trasplante hepático. La resección quirúrgica no es viable si hay enfermedad multifocal, si la reserva funcional hepática es insuficiente o si la proximidad del tumor a estructuras vasculares o biliares impide conseguir márgenes suficientes. ${ }^{4}$ En los últimos 20 años, la ablación local se ha convertido en un tratamiento alternativo importante para el CHC de menor tamaño y casos considerados irresecables mediante cirugía. Se han propuesto y aceptado muchas modalidades diferentes para los procedimientos de ablación; éstos incluyen ablación por radiofrecuencia (RFA), ablación por microondas (MWA), inyección percutánea de etanol (PEI), ablación con láser (LSA), crioablación (CRA), ultrasonido focalizado de alta intensidad (HIFU) y combinaciones de los mismos. ${ }^{3}$ La RFA es utilizada para tratar tumores primarios pequeños $(<5 \mathrm{~cm})$ o recurrentes en pacientes con pobre reserva hepática (Child Pugh B y/o C), y coagulopatía no significativa. La RFA tiene ventajas como ser un método mínimamente invasivo, aplicarse de manera percutánea, utilizarse repetidamente en carcinoma hepatocelular recurrente y ocasionando mínimo daño al parénquima hepáti$\mathrm{CO}$, y baja tasa de complicaciones mayores. ${ }^{5} \mathrm{El}$ objetivo de este estudio es evaluar la eficacia de la RFA en nuestra población.

\section{MATERIAL Y MÉTODOS}

Se realizó un estudio retrospectivo en el que se incluyeron pacientes con diagnóstico histopatológico de $\mathrm{CHC}$ sometidos a RFA, con expediente clínico completo, durante el periodo comprendido del 1 de enero de 2013 al 1 de enero de 2018 en la Unidad Médica de Alta Especialidad del Hospital de Especialidades No. 14, y en el Hospital Regional de Alta Especialidad de Veracruz, México, previa autorización del Comité Local de Ética e Investigación.

Se registraron edad, sexo, índice de masa corporal (IMC), presencia de enfermedad concomitante (diabetes mellitus, hipertensión arterial, hepatitis viral y cirrosis hepática) y valores de laboratorio al momento del diagnóstico: hemoglobina, plaquetas, tiempos de coagulación, International Normalized Ratio (INR), creatinina, alanina aminotransferasa (ALT), aspartato aminotransferasa (AST), fosfatasa alcalina (ALP), bilirrubinas totales, proteínas totales, albúmina y alfa fetoproteína (AFP). Se evaluaron variables correspondientes a la ablación y relacionadas con la lesión (tamaño) y el uso de sorafenib. Los pacientes fueron clasificados según Child-Pugh, ${ }^{6}$ programa italiano del cáncer de hígado (CLIP), ${ }^{7}$ OKUDA, ${ }^{8}$ estadios del cáncer de hígado en la clínica de Barcelona (BCLC), ${ }^{8}$ modelo para la enfermedad hepática terminal (MELD) ${ }^{9}$ e índice albúmina-bilirrubina (ALBI). ${ }^{10}$ Se excluyó a pacientes con diagnóstico final diferente al de $\mathrm{CHC}$ o que no contaran con expediente clínico completo o legible. La supervivencia se calculó a partir del diagnóstico hasta la muerte o la pérdida del seguimiento.

Bajo control ecográfico, se insertó el electrodo StarBurst SDE RFA Device (AngioDynamics, N.Y, USA) en el centro del tumor, el cual fue diseñado para producir ablaciones esféricas en lesiones pequeñas con difícil acceso, aplicando una corriente alterna de alta frecuencia procedente 
del generador. El generador de radiofrecuencia electroquirúrgico utilizado es un modelo 1500X, el cual suministra energía de radiofrecuencia para la coagulación parcial o completa y la ablación de tejido blando, con una potencia de 250 watts combinado con la bomba de infusión de solución salina. Las ondas de radiofrecuencia se administraron durante un tiempo medio de 10 minutos y, tras una pausa, se repitió el procedimiento para asegurar la necrosis.

Análisis estadístico: Se utilizaron media, desviación estándar y rango para describir variables continuas, frecuencias y porcentajes para las variables categóricas. La supervivencia se calculó mediante prueba de Kaplan-Meier y la prueba de Log-Rank (Mantel-Cox) para comparar supervivencia entre grupos. La media estimada de supervivencia se registró en meses. Se realizó curva ROC para determinar área bajo la curva y puntos de corte con sensibilidad y especificidad para predecir aquellos valores que influyen en la supervivencia. Los datos fueron analizados mediante el software SPSS 25 (SPSS, 2016, Chicago, EUA). Un valor de $p<0.05$ se consideró estadísticamente significativo.

\section{RESULTADOS}

Se analizaron 16 pacientes, de los cuales 10 $(62.5 \%)$ fueron mujeres y seis $(37.5 \%)$ hom- bres, la edad media fue de $71.25 \pm 5.8$ años (59-80 años), con IMC promedio de 25.54 $\pm 4.22 \mathrm{~kg} / \mathrm{m}^{2}(17.8-33.7)$. Siete (43.8\%) fueron portadores de diabetes mellitus, cuatro $(25 \%)$ de hipertensión arterial y seis (37.6\%) de hepatitis viral; de estos últimos, cinco $(31.3 \%)$ fueron portadores de hepatitis C y uno (6.3\%) de hepatitis B. Diez (62.5\%) padecieron cirrosis hepática. Los valores de laboratorio se exponen en la Tabla 1. Con base al tipo de ablación, 12 (75\%) fueron abiertas, dos (12.5\%) laparoscópicas y dos (12.5\%) percutáneas. El tamaño promedio de la lesión tumoral fue de $5.81 \pm 2.81 \mathrm{~cm}$ (3-13), de los cuales 10 (62.5\%) tuvieron lesión menor de $5 \mathrm{~cm}$ y seis (37.5\%) lesión mayor de $5 \mathrm{~cm}$. Nueve pacientes (56.3\%) se clasificaron con Child-Pugh A y uno (6.3\%) con Child-Pugh B. La Tabla 2 muestra la estadificación de los pacientes según OKUDA, BCLC, CLIP, MELD y ALBI. Cuatro pacientes (25\%) utilizaron tratamiento con sorafenib y $12(75 \%)$ no lo usaron. Las complicaciones asociadas a RFA ocurrieron en tres casos $(18.75 \%)$ : dos (12.5\%) con falla hepática transitoria y uno $(6.25 \%)$ con neumonía. La media de supervivencia estimada (KaplanMeier) fue de $37.7 \pm 10.2$ meses (IC del 95\%, 17.68-57.84) y la mediana estimada fue de 24 meses. La supervivencia actuarial de todos

Tabla 1: Resultados de laboratorio al momento del diagnóstico de carcinoma hepatocelular.

\section{Parámetro}

Hemoglobina g/dl

Plaquetas $\mathrm{mm}^{3} 10 \mathrm{x}^{9}$

Tiempo de protrombina

International Normalized Ratio

Creatinina mg/dl

ALT UI/l

AST UI/l

Fosfatasa alcalina UI/l

Bilirrubina total mg/dl

Proteínas totales mg/dl

Albúmina g/dl

Alfa fetoproteína

Modelo para la enfermedad hepática terminal

Índice albúmina-bilirrubina

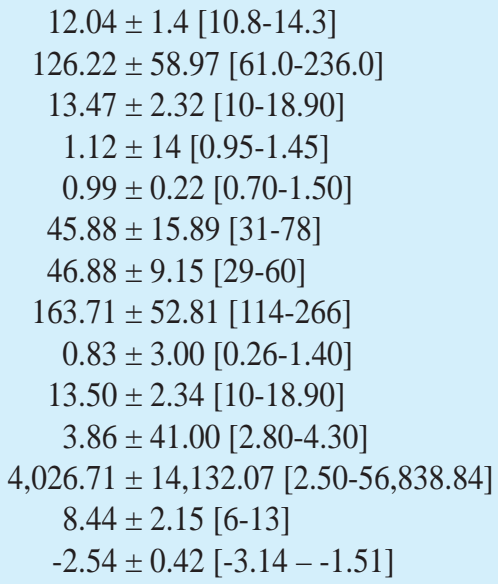




Tabla 2: Estadificación de los pacientes
sometidos a radiofrecuencia.

OKUDA I ninguno positivo, II uno o dos positivos, ALBI Grado 1: $\leq-2.60$, Grado 2: -2.60 a $\leq-1.39$. BCLC = cáncer de hígado en la clínica de Barcelona, CLIP = programa italiano del cáncer de hígado, MELD = modelo para la enfermedad hepática terminal, ALBI = índice albúmina-bilirrubina.

los pacientes a un año fue de $58.5 \%$ y a los cinco años de $23.4 \%$.

Factores asociados a supervivencia. Los pacientes fallecidos tuvieron un menor IMC (23.3 $\left.\pm 3.5 \mathrm{~kg} / \mathrm{m}^{2}\right)$ comparado con los pacientes vivos $\left(27.7 \pm 3.7 \mathrm{~kg} / \mathrm{m}^{2}\right)(\mathrm{p}=0.031$, $\mathrm{t}$ de Student). Ninguna de las otras variables registradas tuvo diferencia significativa en la supervivencia de los pacientes como se muestra en la Tabla 3. Se realizó análisis mediante curva ROC del IMC, encontrando un área bajo la curva de 0.79 (95\%, IC 0.56-1.0) ( $p=0.046)$ con un punto de corte de 22.06 (sensibilidad del 100\%, especificidad $38 \%)$. Sin embargo, no existió diferencia en la supervivencia estimada por Kaplan-Meier (LogRank) al aplicar el punto de corte $<22.06 \mathrm{~kg} /$ $\mathrm{m}^{2}$ con media $16.0 \pm 10.53$ meses (0.0-36.6) $y>22.06 \mathrm{~kg} / \mathrm{m}^{2}$ con media $47.4 \pm 12.2$ meses (23.5-71.3) у $\mathrm{p}=0.20$.

\section{DISCUSIÓN}

Los resultados de nuestro estudio indican que los pacientes sometidos a RFA tienen menor supervivencia a un año y a cinco años, comparado con otras publicaciones que aplicaron el mismo tratamiento. No obstante, encontramos que casi $40 \%$ de las lesiones eran mayores de $5 \mathrm{~cm}$ y que la mayoría de los pacientes se encontraban en estadios más avanzados que los recomendados por la BCLC para realizar RFA. Existen estudios con supervivencia superior a nuestros resultados cuando los pacientes presentan lesiones menores de $5 \mathrm{~cm}$. Lencioni y colaboradores ${ }^{11}$ evaluaron pacientes cirróticos (Child-Pugh A y/o B), estadio temprano de CHC (BCLC A, lesiones menores a $5 \mathrm{~cm}$ ) sometidos a RFA, obteniendo una supervivencia de $97 \%$ al primer año y de $41 \%$ a los cinco años, con una mediana de 49 meses. ${ }^{11}$ Kim y su grupo, ${ }^{12}$ en pacientes sometidos a RFA, obtuvieron supervivencia de $59.7 \%$ a cinco años, siendo sus predictores significativos para baja supervivencia: la edad del paciente, Child-Pugh B y la presencia de recurrencia extrahepática. A su vez, Lee y asociados ${ }^{13}$ evaluaron 162 pacientes con más de tres tumores con un diámetro máximo de $5 \mathrm{~cm}$, encontrando una supervivencia de $67.9 \%$ a cinco años, donde los predictores más significativos de baja supervivencia fueron Child-Pugh B, niveles elevados de AFP y presencia de hipertensión portal. Pese a que estos estudios registraron mayor supervivencia que el nuestro, existen otros en los que la supervivencia es parecida a la nuestra, en donde las lesiones son mayores a $5 \mathrm{~cm}$ y se encuentran en estadios intermedios de BCLC. Entre estos estudios se encuentran el de Nouso K y colaboradores $^{14}$ que incluyó 91 pacientes en estadios intermedios de BCLC (B1, B2 y B3) y en el que se logró supervivencia de $73.8 \%$ a tres años y $57.3 \%$ a cinco años; el de Dai W y asociados, ${ }^{14}$ que involucró a 63 pacientes sometidos a RFA con tamaño promedio de la lesión de $6.0 \mathrm{~cm}$ (5.2-8.0), obteniendo supervivencia a uno, tres y cinco años de $93.3,70.5$ y $20.9 \%$, respectivamente, con una mediana de supervivencia de 39.8 meses; $y$ finalmente el de Yin X y colaboradores ${ }^{15}$ que analizó 49 casos con tamaño tumoral entre 3-7 cm con supervivencia a un año de $75.8 \%$ y a cinco años de $15.4 \%$. Este 
uso de la RFA en estadios intermedios y lesiones mayores de $5 \mathrm{~cm}$ con supervivencias a cinco años similares a la nuestras (alrededor de 20\%) puede justificar la utilización de este recurso en nuestra población, mismo que inició y fue reportado desde el año $2006 .^{16}$

Existen estudios que apoyan que el IMC tiene un impacto significativo en el pronóstico de pacientes con CHC. Qinggan Li y colaboradores $^{17}$ analizaron 379 pacientes, donde aquellos con IMC menor a $23 \mathrm{~kg} / \mathrm{m}^{2}$ tuvieron una supervivencia de 353.9 días (316.9-391.0) e IMC mayor a $23 \mathrm{~kg} / \mathrm{m}^{2}$ una supervivencia de
571.8 días (532.3-611.4). Asimismo, Xiyu Liu y asociados ${ }^{18}$ evaluaron 136 pacientes, obteniendo mayor supervivencia en IMC menor a $25 \mathrm{~kg} / \mathrm{m}^{2}$ de $95 \%$ al año y $16 \%$ a los cinco años que en aquellos con IMC mayor a $25 \mathrm{~kg} / \mathrm{m}^{2}(\mathrm{p}$ $=0.048)$. Nuestro estudio refleja una media estimada de supervivencia de 1,410 días (47 meses) en pacientes con IMC mayor a $22.06 \mathrm{~kg} /$ $\mathrm{m}^{2}$; además, estos pacientes sí alcanzaron registros de supervivencia superiores a cinco años. Desafortunadamente no pudimos confirmar esta asociación como factor de riesgo mediante análisis estadístico (curva ROC/regresión de

\section{Tabla 3: Estimaciones de la supervivencia por el método de Kaplan-Meier.}

\begin{tabular}{|c|c|c|c|c|c|}
\hline Variable & $\begin{array}{c}\text { Media } \pm \mathrm{DE} \\
\text { meses }\end{array}$ & IC $95 \%$ & $\begin{array}{l}\text { Supervivencia } \\
\text { a un año (\%) }\end{array}$ & $\begin{array}{l}\text { Supervivencia } \\
\text { a cinco años } \\
(\%)\end{array}$ & $\mathbf{p}$ \\
\hline \multicolumn{6}{|c|}{ Índice de masa corporal } \\
\hline$<22.06$ & $16.00 \pm 10.53$ & $0-36.65$ & 34.17 & NR & 0.2 \\
\hline$>22.06$ & $47.43 \pm 12.2$ & $23.51-71.35$ & 64.70 & 48.55 & \\
\hline \multicolumn{6}{|c|}{ Diabetes mellitus } \\
\hline No & $44.50 \pm 15.66$ & $13.79-75.2$ & 66.45 & 44.40 & 0.73 \\
\hline Sí & $23.31 \pm 5.7$ & $12.13-34.49$ & 53.33 & NR & \\
\hline \multicolumn{6}{|c|}{ Hipertensión arterial } \\
\hline No & $41.94 \pm 13.7$ & $15.08-68.80$ & 83.30 & 27.78 & 0.28 \\
\hline Sí & $14.31 \pm 3.22$ & 8-20.63 & 56.14 & NR & \\
\hline \multicolumn{6}{|c|}{ Hepatitis viral } \\
\hline No & $37.93 \pm 13.00$ & $12.43-63.42$ & 66.66 & 35.56 & 0.91 \\
\hline Sí & $26.16 \pm 7.69$ & $11.08-41.24$ & 66.67 & NR & \\
\hline \multicolumn{6}{|c|}{ Cirrosis hepática } \\
\hline No & $15.66 \pm 4.04$ & 7.74-23.59 & 67.50 & 33.75 & 0.82 \\
\hline Sí & $33.83 \pm 10.07$ & $14.09-53.57$ & 66.10 & NR & \\
\hline \multicolumn{6}{|c|}{ Tamaño de lesión } \\
\hline$<5 \mathrm{~cm}$ & $21.75 \pm 6.08$ & 9.83-33.66 & 53.57 & NR & 0.25 \\
\hline$>5 \mathrm{~cm}$ & $51.42 \pm 14.03$ & 23.92-78.92 & 71.43 & 53.57 & \\
\hline \multicolumn{6}{|l|}{ OKUDA } \\
\hline I & $40.42 \pm 11.82$ & $17.25-63.59$ & 72.92 & 27.34 & 0.43 \\
\hline II & $14.16 \pm 4.05$ & $6.21-22.12$ & 50.00 & NR & \\
\hline \multicolumn{6}{|c|}{ Alfa fetoproteína } \\
\hline$<200$ & $34.37 \pm 11.2$ & $12.40-56.34$ & 60.61 & 22.73 & 0.68 \\
\hline$>200$ & $14.25 \pm 4.95$ & 4.53-23.96 & 50.00 & NR & \\
\hline \multicolumn{6}{|l|}{ Sorafenib } \\
\hline No & $20.00 \pm 3.68$ & $12.78-27.21$ & 66.45 & NR & 0.93 \\
\hline Sí & $38.04 \pm 12.45$ & $13.63-62.44$ & 58.74 & 29.37 & \\
\hline
\end{tabular}

OKUDA I ningún criterio positivo, II uno o dos positivos, DE = desviación estándar, NR = no registrado. 
Cox), posiblemente por el tamaño de nuestra muestra. Nuestro estudio apunta que el uso de sorafenib posterior a la RFA influye en una mayor supervivencia que en aquellos pacientes que no lo utilizaron. Esto está de acuerdo con la serie de Feng y colaboradores ${ }^{19}$ que incluyó 64 pacientes con terapia única (RFA) y 64 con terapia combinada (RFA y sorafenib), encontrando una media de supervivencia de 118.6 semanas y 161.8 semanas, respectivamente; y con el estudio de Quanyou y asociados ${ }^{20}$ que analizó 50 pacientes con terapia única y 40 con terapia combinada, encontrando una supervivencia libre de tumor de 8.4 y 12.3 meses, respectivamente, con una media de seguimiento de 35 meses.

Existe muy poca información sobre la utilización de RFA en pacientes con $\mathrm{CHC}$ en nuestro país. Mondragón-Sánchez y colaboradores ${ }^{21}$ publicaron la utilización de RFA en una variedad de tumores hepáticos, siendo la mayoría $\mathrm{CHC}(\mathrm{n}=18)$ con un tamaño promedio de lesión de $5.5 \mathrm{~cm}$ con una supervivencia a un año alrededor de $20 \%$ y una media estimada de 18 meses. Posteriormente, Ladrón de Guevara y asociados $^{22}$ publicaron el uso de RFA en dos pacientes con $\mathrm{CHC}$ con una supervivencia de un año de 40 y ocho meses promedio estimado de supervivencia. Recientemente, Cisneros Garza y colaboradores ${ }^{23}$ señalaron el uso de RFA en 13 pacientes, seis en estadio A, siete en estadio $B$ y uno en estadio $C$ sin señalar específicamente la supervivencia de éstos. Los resultados de nuestro estudio evidenciaron una mejor supervivencia tanto en promedio estimado como porcentaje de supervivencia a un año sin poder compararlo a cinco años, dado que no fue reportado, por lo cual asumimos que este es el estudio mexicano de RFA por $\mathrm{CHC}$ con mayor seguimiento promedio publicado hasta ahora.

\section{CONCLUSIONES}

La RFA en Veracruz, México, es utilizada en pacientes con $\mathrm{CHC}$ en estadios variados y en lesiones tumorales de diversas dimensiones, con resultados mixtos, dependiendo de las características a estudiar. Los resultados de la RFA en Veracruz, México, puede ser el mayor seguimiento de esta terapéutica en nuestro país. Dado el tamaño de la muestra, no se puede establecer factores de riesgo que influyan significativamente en esta terapia.

\section{REFERENCIAS}

1. Wallace M, Preen D, Jeffrey G, et al. The evolving epidemiology of hepatocellular carcinoma: a global perspective. Expert Rev Gastroenterol Hepatol. 2015; 9: 765-779.

2. Jemal A, Bray F, Center MM, et al. Global cancer statistics. CA Cancer J Clin. 2011; 61: 69-90.

3. Verslype C, Rosmorduc O, Rougier P. Hepatocellular carcinoma: ESMO-ESDO Clinical Practice Guidelines for diagnosis, treatment and follow-up. Ann Oncol. 2012; 23: vii43-vii45.

4. Wong WS, Patel SC, Cruz FS, et al. Cryosurgery as a treatment for advanced stage hepatocellular carcinoma: results, complications, and alcohol ablation. Cancer. 1998; 82: 1268-1278.

5. Ning Chong CC, San Lai P. Treatment strategy for recurrent hepatocellular carcinoma. Hong Kong; 2012. 123-126. [cited 27 February 2019]. Available from: https://www.intechopen.com/download/pdf/27581

6. Song T, Wai Kit Ip E, Fong Y. Hepatocellular carcinoma: current surgical management. Gastroenterology. 2004; 127: S248-S260.

7. Kudo M, Chung H, Osaki Y. Prognostic staging system for hepatocellular carcinoma (CLIP score): its value and limitations, and a proposal for a new staging system, the Japan Integrated Staging Score (JIS score). J Gastroenterol. 2003; 38: 207-215.

8. Adhoute X, Pénaranda G, Raoul J, et al. Barcelona clinic liver cancer nomogram and others staging/scoring systems in a French hepatocellular carcinoma cohort. World J Gastroenterol. 2017; 23: 2545-2555.

9. Kamath PS, Kim WR. The model for end-stage liver disease (MELD). Hepatology. 2007; 45: 797-805.

10. Chen B, Lin S. Albumin-bilirubin (ALBI) score at admission predicts possible outcomes in patients with acute-on-chronic liver failure. Medicine. 2017; 9: 1-5.

11. Lencioni R, Cioni D, Crocetti L, et al. Early-stage hepatocellular carcinoma in patients with cirrhosis: long-term results of percutaneous image-guided radiofrequency ablation. Radiology. 2005; 234: 961-967.

12. Kim YS, Lim HK, Rhim H, et al. Ten-year outcomes of percutaneous radiofrequency ablation as first-line therapy of early hepatocellular carcinoma: analysis of prognostic factors. J Hepatol. 2013; 58: 89-97.

13. Nouso K, Kariyama K, Nakamura S, et al. Application of radiofrequency ablation for the treatment of intermediate-stage hepatocellular carcinoma. J Gastroenterol Hepatol. 2017; 32: 695-700.

14. Dai W, Cheung T, Chok K, et al. Radiofrequency ablation versus transarterial chemoembolization for unresectable solitary hepatocellular carcinomas sized 5-8 cm. HPB (Oxford). 2015; 17: 226-231.

15. Yin $X, X i e X$, Lu M, et al. Percutaneous thermal ablation of medium and large hepatocellular carcinoma. Cancer. 2009; 115: 1914-1923.

16. Mier GM, Torres SE. Clinical images in gastroenterology. Radiofrequency ablation of the 
right lobe hepatocarcinoma. Rev Gastroenterol Mex. 2006; 71: 508.

17. Li Q, Xing H, Liu D, Li H. Negative impact of low body mass index on liver cirrhosis patients with hepatocellular carcinoma. World J Surg Oncol. 2015; 13: 1-4.

18. Liu X, Xu J. Body mass index and waistline are predictors of survival for hepatocellular carcinoma after hepatectomy. Med Sci Monit. 2015; 21: 2203-2209.

19. Feng X, Xu R, Du X, et al. Combination therapy with sorafenib and radiofrequency ablation for BCLC Stage 0-B1 hepatocellular carcinoma: a multicenter retrospective cohort study. Am J Gastroenterol. 2014; 109: 1891-1895.

20. Gong Q, Qin Z, Hou F. Improved treatment of early small hepatocellular carcinoma using sorafenib in combination with radiofrequency ablation. Oncol Lett. 2017; 14: 7045-7048.

21. Mondragón-Sánchez R, Murrieta-González H, Martínez-González MN, et al. Ablation of malignant liver tumors with radiofre- quency. A series of cases in Mexico. Rev Gastroenterol Mex. 2009; 74: 212216.

22. Ladron de Guevara L, Rojas-Macuil P, Sanchez-Chavez $X$, et al. Hepatocellular carcinoma: epidemiological profile from a cohort of federal employees in Mexico. Ann Hepatol. 2009; 8: 212-219.

23. Cisneros Garza LE. Characterization of hepatocellular carcinoma in Mexico. Rev Gastroenterol Mex. 2018; 83: $1-5$

Consideraciones y responsabilidad ética: El estudio fue aprobado previamente por el Comité de Ética e Investigación Institucional Local. Los autores declaran que este artículo no contiene información personal que permita identificar a los pacientes. Financiamiento: Recursos del Hospital donde se realizó el estudio.

Conflicto de intereses: Sin conflicto de intereses.

Correspondencia:

Gustavo Martínez Mier MD

Corporativo San Gabriel

Calle Alacio Pérez Núm. 918-314,

Col. Ignacio Zaragoza, 91910,

Veracruz, Ver., México.

E-mail: gmtzmier@gmail.com, gmtzmier@hotmail.com 